\title{
Tanzanie: les Wagogo, maîtres de la musique
}

\section{Emmanuelle Olivier}

\section{OpenEdition}

\section{Journals}

Édition électronique

URL : http://journals.openedition.org/ethnomusicologie/641

ISSN : 2235-7688

Éditeur

ADEM - Ateliers d'ethnomusicologie

Édition imprimée

Date de publication : 1 novembre 2003

Pagination : 260-262

ISBN : 978-2-8257-0863-7

ISSN : 1662-372X

Référence électronique

Emmanuelle Olivier, «Tanzanie: les Wagogo, maîtres de la musique », Cahiers d'ethnomusicologie [En ligne], 16 | 2003, mis en ligne le 16 janvier 2012, consulté le 01 mai 2019. URL : http:// journals.openedition.org/ethnomusicologie/641

Ce document a été généré automatiquement le 1 mai 2019.

Tous droits réservés 


\title{
Tanzanie: les Wagogo, maîtres de la musique
}

\author{
Emmanuelle Olivier
}

\section{RÉFÉRENCE}

Tanzanie. Chants wagogo, Paris: Ocora-Radio France, C560155, 2000

Tanzanie. Musiques rituelles Gogo, Genève: AIMP LXVI, VDE CD-1067, 2001

Tanzanie. Musique de divertissement wagogo, Paris: Ocora-Radio France, C560165, 2002,

Enregistrements, livret et photos de Polo Vallejo

1 À travers trois CDs, Polo Vallejo nous propose une véritable anthologie de la musique wagogo , dont on ne mesurait pas jusqu'à présent la richesse. Les enregistrements, clairs et chaleureux, révèlent une musique polyphonique impressionnante de diversité et de complexité, mais aussi de beauté, tandis que les livrets nous guident dans la compréhension de cette culture musicale par une analyse des outils (instruments et voix), du système proprement dit et des modalités de performance, ainsi que des paroles des chants et du contexte social. Quant aux photos, elles sont ici bien plus qu'un support commercial: elles donnent à voir avec une force empreinte de sensibilité des hommes et des femmes qui conçoivent leur musique comme un moment essentiel de leur vie.

2 Les trois CDs illustrent, chacun dans un registre particulier, le riche éventail des musiques wagogo. Le premier (CD 1) est essentiellement consacré à la polyphonie vocale masculine exécutée lors des rituels de récolte et d'initiation. Le deuxième (CD 2) nous fait découvrir les grands chants polyphoniques de femmes liés aux deux mêmes rituels, au mariage et aux funérailles, mais qui servent également à guérir le bétail, à formuler des «reproches à l'encontre de ceux qui ne respectent pas les normes de la vie communautaire» et à rétablir l'équilibre après un problème qui menace la communauté. Enfin, le troisième $\mathrm{CD}$ ( $\mathrm{CD} 3$ ) est entièrement dédié aux instruments mélodiques qui 
soutiennent les chants de divertissement ou les chants rituels exécutés dans des contextes plus spontanés.

3 Ce qui frappe immédiatement, c'est la diversité des procédés polyphoniques réunis en une même musique: hétérophonie, bourdon, mouvements parallèles, homophonie, hoquet, canon simple ou entrelacé, imitation et contrepoint tantôt alternent tantôt se superposent de manière virtuose, comme si les Wagogo en jouaient avec une véritable jubilation. On ne peut être que séduit par la performance de deux chœurs homophones, l'un masculin, l'autre féminin, qui chantent en contrepoint, alternés au seul chœur masculin en hoquet (CD 1, plage 8). De plus, le passage d'un procédé polyphonique à un autre est annoncé par un instrument ou par un signal vocal, témoignant là d'une architecture formelle sophistiquée et parfaitement maîtrisée.

4 Mais au-delà, c'est l'ensemble du langage musical qui mérite notre attention d'ethnomusicologue, en ce que la plupart de ses traits sont à la fois originaux et complexes au regard des musiques d'Afrique subsaharienne.

5 Ainsi, comme nombre d'autres populations africaines, les Wagogo privilégient une échelle pentatonique anhémitonique, mais qui comporte ici un triton (ré $f a$ sol la si) plus exceptionnel (bien que présent dans certaines musiques khoisan d'Afrique australe), utilisé en intervalle mélodique et harmonique pour donner une couleur particulière et, sur un plan syntaxique, pour signifier la fin des sections homophoniques. Mais l'aspect le plus remarquable est qu'hommes et femmes chantent simultanément dans des modes pentatoniques différents, ce qui complexifie d'autant la texture polyphonique.

6 La musique est mesurée et périodique et, si les périodes comptent un nombre pair de pulsations, caractéristique tout à fait commune en Afrique subsaharienne, ce nombre peut aussi être impair, quoique plus rarement. De plus, les Wagogo aiment à brouiller leurs repères métriques, non pas seulement en augmentant proportionnellement la durée de la période (de 8 pulsations, par exemple, on passerait à 16, 24 ou 32), mais en réalisant des périodes irrégulières dont les durées ne sont pas proportionnelles et en inversant les segments musicaux (CD 1, plage 12): autant de procédés peu observés ailleurs qu'en Afrique australe.

7 Quant au rythme, on est évidemment plus sensible à ses ruptures au sein d'une pièce, créant ainsi des changements de dynamique ( $\mathrm{CD} 3$, plage 4) qu'à sa complexité (superposition de plusieurs figures en polyrythmie), par ailleurs omniprésente en Afrique.

8 L'ensemble de ces traits est investi principalement dans des chants, la voix étant, comme le remarque Polo Vallejo, l'outil par excellence des Wagogo, utilisée selon des techniques très diversifiées, allant des halètements au yodel en passant par les cris, les glissandi, et les sons tremblés, et dont le timbre est également travaillé (voix déguisée avec de petits bourdonnements et usage du vibrato). Mais les chants peuvent aussi être soutenus par des instruments rythmiques et mélodiques dont la diversité est une fois de plus surprenante, toutes les familles étant représentées: idiophones (lamellophones, xylophones, hochets, sistre), cordophones (vièle à 2 ou 3 cordes, cithare à 13 cordes avec double caisse de résonance, arc musical à résonateur buccal ou avec calebasserésonateur), aérophones (sifflet, cornes, flûte traversière) et membranophones (tambours).

9 Autre élément remarquable: l'esthétique de la musique instrumentale, extrêmement raffinée. Les Wagogo choisissent chaque matériau en fonction de sa sonorité particulière, 
tandis que le facteur accorde son instrument selon une échelle non tempérée pour produire une certaine épaisseur lorsque voix (basées sur une échelle tempérée) et instruments se superposent. Enfin, lors des performances collectives, chaque instrument a un rôle précis, ce qui contribue à créer une couleur et un relief sonores particuliers.

Mais le talent de Polo Vallejo ne se réduit pas à la découverte et à l'analyse d'une musique exceptionnelle: il nous en dévoile également la dynamique. En proposant plusieurs versions d'une même pièce, l'auteur nous invite à saisir comment les Wagogo transposent un chant polyphonique aux instruments ( $\mathrm{CD} 2$, plages 1,$2 ; \mathrm{CD} 3$, plages $6,9,10)$, et, à l'inverse, comment ils imitent les instruments avec leurs voix (CD 3, plage 17), mais aussi comment instruments et voix parviennent à une véritable symbiose ( $\mathrm{CD} 3$, plage 7: vièle grave et voix de l'instrumentiste qui imite la vièle aiguë). L'auteur nous fait aussi participer à une situation d'apprentissage, par l'écoute d'une même pièce chantée par les adultes, puis reprise par les enfants (CD 1, plages 21, 22).

11 Polo Vallejo aborde également la dynamique sociale de la musique, en mentionnant, peutêtre trop brièvement, les processus de disparition, de transformation et de création de pièces. Sa contribution est telle que nous aimerions presque en savoir plus: en quoi et par qui la musique se renouvelle, par exemple. L'auteur fait remarquer que la proximité et l'influence de la ville conduisent à une «acculturation», mais selon quels processus et surtout que définit-on exactement par ce terme? la culture n'est-elle pas en soi déjà une acculturation? Les Wagogo renoncent-ils tout bonnement à leur musique ou la transforment-ils au contact d'autres musiques, et lesquelles? Le syncrétisme religieux, dont l'importance a souvent été soulignée pour l'Afrique centrale, a-t-il également des conséquences sur les pratiques musicales, voire le système proprement dit? De même, les données à propos des interactions avec les autres groupes wagogo sont peut-être à développer: on ne sait en quoi elles consistent et quelles répercussions elles ont sur la musique du groupe étudié. Autant de questions qui restent en suspens, même si nous avons bien conscience que les livrets de CDs ne sont pas le lieu privilégié pour analyser en profondeur, et dans toute leur complexité, de tels phénomènes.

Enfin, dans une perspective comparatiste, ces enregistrements et la première analyse qu'en propose Polo Vallejo nous permettent désormais de mettre en regard les chants contrapuntiques wagogo avec d'autres polyphonies vocales africaines du même type, comme celles des Pygmées centrafricains, des Bushmen du Kalahari ou des Dorze éthiopiens. Nombre de questions se posent alors parmi lesquelles celle de savoir si des musiques qui, à l'audition, semblent extrêmement proches, sont conçues et élaborées de la même manière par leurs détenteurs. En d'autres termes, analyser ces polyphonies c'est se demander comment caractériser l'objet musical: est-ce par ses techniques de production ou par le cheminement mis en œuvre pour aboutir à cet objet? Autant dire que nous attendons avec impatience les prochaines publications de Polo Vallejo sur ces extraordinaires musiques wagogo. 